\title{
Analysis of the behavior and 2D modeling of ferrite inductors with $E$ geometry: comparison with 3D model and real measurements
}

\author{
R. A. Salas ${ }^{1}$ and J. Pleite ${ }^{1}$ \\ ${ }^{1}$ Departamento de Tecnología Electrónica, Escuela Politécnica Superior, Universidad Carlos III de Madrid, Avda. de la \\ Universidad, 30, 28911 Leganés (Madrid), Spain
}

\begin{abstract}
In this paper we propose and validate by experiment a practical method to compute by simulations the inductance of a ferrite inductor as a function of the current intensity level from the linear to the saturation regions. Our method combines the use of 2D Finite Element Analysis with experimental measurements taken in a laboratory environment. We also present and analyze results of the convergence and computational cost of the $2 \mathrm{D}$ and $3 \mathrm{D}$ simulations showing the reduction of computational cost when we chose the 2D simulation.
\end{abstract}

\section{Introduction}

Ferrites are an ideal core material for transformers and inductors due to the combination of low core cost and low core losses [1]. We can find a great variety of shapes and sizes. In particular E cores are popular shapes due to their lower cost, ease of assembly and their winding [2]. In order to carry out precise simulations of a ferrite inductor it is necessary to include the nonlinear behavior of the core [3-5] and the geometrical effects in the model. Our aim is the modeling of E-type ferrite inductors (Figure 1(a)) by means of Finite Element Analysis (FEA). Until now investigations of this core have been mainly oriented towards the study of the windings and the effects of frequency and 3D geometry, considering a linear behavior of the core [6-7].

In this paper we propose a specific modeling procedure for the simulation in 2D of an inductor with an E type ferrite core. The procedure is capable of reproducing both the nonlinear magnetic flux-current curve and the corresponding inductance-current curve in the ferrite's complete working range, from the linear to the saturation region. The Finite Element simulations are carried out in $2 \mathrm{D}$ with its advantages being considerable reduction of the computational cost as well as being able to achieve convergence. We report results of a preliminary study of the convergence and of the computational cost and report results of the flux values and their corresponding inductances, both expressed as a function of the excitation current applied to the inductor. We test the accuracy of the method by comparing the experimental data with those computed by FEA simulations. We also present an experimental study on how the variation of the number of turns of the inductor influences both the magnetic flux and the inductance.

\section{Measuring method and computation method by Finite Elements}

In this section we will focus on describing the measuring method and the computation method by Finite Elements we have used. The measuring method has three main objectives:

The first objective is to analyze the nonlinear behavior of the ferrite cores in all working regions (linear, intermediate and saturation regions), and in particular to analyze the influence of both the excitation level and the air-gap thickness on the values of the magnetic flux and the inductance as a function of the current used. The second objective is to analyze both the influence of the number of turns of the inductor and the influence of the size of the ferrite core. The third one is to validate the procedures carried out through the comparison of the obtained results with experimental measurements.

Accordingly we build two two-winding transformers and take measurements under DC current. One of the transformers has a toroidal core and the other one an E-type core. By using the toroidal transformer we measure the magnetic flux as a function of the DC excitation current I ( $\Phi$-/ curve) and derive from this the nonlinear $B-H$ curve (first magnetization curve) that characterizes the core material. The $B-H$ curve is an input parameter in the simulations by Finite Element Analysis. In order to measure the $\Phi$-/ curve we apply a DC current to the primary winding of the transformer and measure the flux in the secondary winding with a fluxmeter. By using the E-transformer we measure the $\Phi$-/ curve and derive from it inductance values ( $L-/$ curve) by differentiating numerically $\Phi$ with respect to

This is an Open Access article distributed under the terms of the Creative Commons Attribution License 2.0, which permits unrestricted use, distribution, and reproduction in any medium, provided the original work is properly cited. 
I. The experimental $L-/$ curve is necessary to validate the $L-/$ curve computed by Finite Element simulations.

Once we have finished the measuring procedure we start with the modeling procedure by FEA with the magnetostatic solver of the Ansoft Maxwell software. In addition to the 2D inductor model (cross-section of the real inductor), it has as main inputs the experimental $B-H$ curve, the values of the DC excitation current from the linear to the saturation regions and the parameters to generate the mesh. To generate the mesh in $2 \mathrm{D}$, we have chosen an adaptative refinement of the mesh which consists in making a finer mesh at the spatial points that are more irregular, such as: corners, regions with irregular borders, etc. This technique reduces the computing time and the convergence and tolerance, and this algorithm is implemented into the Maxwell program. We then specify the parameters related to the adaptative analysis to generate the mesh: percent refinement per pass, the number of requested passes to stop the algorithm and the percent error $(\tau)$. The simulation program gives, as results for each value of $/$, the spatial distribution of the $B$ field on the inductor.

To obtain the $\Phi$-/ curve, for each value of /, we compute $\Phi$ by numerical integration of the normal induction component taken over a cross-section in the core, which can be chosen in a lateral column or in the central post. As the ferrite is characterized by a high relative permeability $\left(\mu_{r} \approx\right.$ $2000)$ in comparison to vacuum $\left(\mu_{r} \approx 1\right)$ the leakage flux is negligible. From the $\Phi-/$ curve we derive the $L-/$ curve by differentiating $\Phi$ with respect to $/$.

In order to validate the procedure we compare $2 \mathrm{D}$ results with 3D and experimental results.

\section{Simulation and modeling results and experimental validation}

The E-type ferrite inductor consists of two identical halves, a winding of copper wire and a coil former. In order to model the inductor we carry out $3 \mathrm{D}$ and $2 \mathrm{D}$ numerical simulations by means of Finite Element Analysis with the magnetostatic solver of the Ansoft Maxwell field simulator run on a personal computer. In Figure 1 we show the real inductor and its corresponding 3D model. In the first place, we run simulations in $3 \mathrm{D}$ with the objective of checking if there is initial convergence in $3 \mathrm{D}$ for four inductors with different sizes of the E-type core and a different number of turns (Table 1). In particular, we choose E-type ferrite cores from the Ferroxcube manufacturer made of $3 \mathrm{~F} 3$ material, a MnZn soft ferrite material.

Table 1. Tested inductors.

\begin{tabular}{|c|c|}
\hline Ferrite Core type & Number of turns \\
\hline E65/32/27 & 45 \\
\hline E47/20/16 & 28 \\
\hline E34/14/9 & 28 \\
\hline E20/10/5 & 15 \\
\hline
\end{tabular}

After running the program for approximately 15 hours we found that in the case of the three biggest cores we were unable to achieve convergence with or without the coil former or even by simplifying the geometry (e.g. right- angled corners instead of rounded corners) with the message "insufficient memory for module hnl3d" showing the nonconvergence of the procedure. We only found 3D convergence in the case of the smallest core (E20/10/5). Next, with this core we study the influence of the air-gap $g$ on the computational cost.

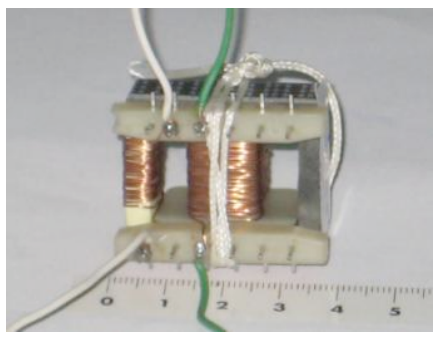

(a)

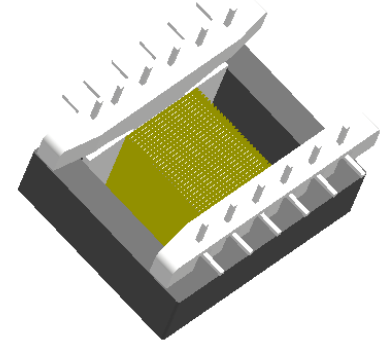

(b)
Fig. 1. (a) Real inductor. (b) 3D model.

For this purpose we explored the cases where $g=0$ (ungapped) and $g>0$, choosing for this latter case the value of $g=200 \mu \mathrm{m}$. For each value of the air-gap to be studied ( $g$ $=0$ and $g=200 \mu \mathrm{m}$ ), we carried out 3D simulations with different values of excitation currents: $/=0.01,0.02,3,8$, 10 and $20 \mathrm{~A}$. The values $/=0.01$ and $0.02 \mathrm{~A}$ are in the linear region and $I=3,8,10$ and $20 \mathrm{~A}$ are in the saturation region. We present an example of our numerical experiments in Tables 2 and 3, where we show that the introduction of the air-gap requires a greater number of finite elements and a higher computational cost.

Table 2. Computational cost in the 3D simulations for the ungapped case.

\begin{tabular}{|c|c|c|}
\hline & \multicolumn{2}{|c|}{$g=0 \mu \mathrm{m}$} \\
\hline$I(\mathrm{~A})$ & CPU time & Tetrahedra \\
\hline 0.01 & 2 min. 59 s & 77280 \\
\hline 0.02 & 4 min. 44 s & 87327 \\
\hline 3 & 16 min. 52 s & 87327 \\
\hline 8 & 14 min. 24 s & 87327 \\
\hline 10 & 13 min. 19 s & 87327 \\
\hline 20 & 11 min. 46 s & 87327 \\
\hline
\end{tabular}

Table 3. Computational cost in the $3 \mathrm{D}$ simulations for the case with gap.

\begin{tabular}{|c|c|c|}
\hline & \multicolumn{2}{|c|}{$g=200 \mu \mathrm{m}$} \\
\hline$/(\mathrm{A})$ & CPU time & Tetrahedra \\
\hline 0.01 & $11 \mathrm{~min} .58 \mathrm{~s}$ & 250042 \\
\hline 0.02 & $11 \mathrm{~min} .59 \mathrm{~s}$ & 250042 \\
\hline 3 & 24 min. $46 \mathrm{~s}$ & 250042 \\
\hline 8 & 33 min. $28 \mathrm{~s}$ & 250042 \\
\hline 10 & 52 min. $22 \mathrm{~s}$ & 250042 \\
\hline 20 & 1 h. 8 min. & 250042 \\
\hline
\end{tabular}

Once we completed the $3 \mathrm{D}$ calculation process we started the $2 \mathrm{D}$ simulations with the same objectives as in $3 \mathrm{D}$ : to check the convergence and to investigate the influence of the air-gap on the computational cost. For this purpose, we performed simulations in 2D using cross-sections in the $x y$ plane of the aforementioned four inductors. These cross- 
sections are included in rectangular or square computational domains. We perform simulations for different values of $\tau$ $(0.2,0.5$ and $1 \%$ ); for each $\tau$ we start by simulating the ungapped inductor $(g=0)$ with different excitation currents $I=0.01$ A (linear region), 0.02 A (linear region), 3, 8 and 20 A (saturation region). Once this is finished, we repeat all of these calculations for each value of $g$ using gapped cores. We use the values of $g=2,4,6,8,10,20, \ldots, 100,150$ and $200 \mu \mathrm{m}$ and achieve convergence in all cases. In Tables 4 and 5 we summarize the computational cost for two values of $g$ and two sizes of the core for different values of excitation current /. By using the design of the 2D E-type inductor (cross-section of the real inductor) we find convergence in the 2D simulations for all explored sizes of the core and gap-thicknesses. The number of finite elements remains constant at different excitation currents and, as expected, increases with growing core sizes.

Table 4. Computational cost in the $2 \mathrm{D}$ simulations.

\begin{tabular}{|c|c|c|c|c|}
\hline & \multicolumn{3}{|c|}{ E65/32/27 core } \\
\hline & \multicolumn{2}{|c|}{$\mathrm{g}=0 \mu \mathrm{m}$} & \multicolumn{2}{c|}{$\mathrm{g}=200 \mu \mathrm{m}$} \\
\hline$/(\mathrm{A})$ & Time $(\mathrm{s})$ & Elements & Time $(\mathrm{s})$ & Elements \\
\hline 0.01 & 16 & 10776 & 10 & 9336 \\
\hline 20 & 18 & 10776 & 9 & 9336 \\
\hline
\end{tabular}

Table 5. Computational cost in the $2 \mathrm{D}$ simulations.

\begin{tabular}{|c|c|c|c|c|}
\hline & \multicolumn{3}{|c|}{ E34/14/9 core } \\
\hline & \multicolumn{2}{|c|}{$\mathrm{g}=0 \mu \mathrm{m}$} & \multicolumn{2}{c|}{$\mathrm{g}=200 \mu \mathrm{m}$} \\
\hline$/(\mathrm{A})$ & Time $(\mathrm{s})$ & Elements & Time $(\mathrm{s})$ & Elements \\
\hline 0.01 & 8 & 6256 & 6 & 5919 \\
\hline 20 & 10 & 6256 & 6 & 5919 \\
\hline
\end{tabular}

In Figure 2(a) we show the mesh generated by the program in a $2 \mathrm{D}$ simulation using the E47/20/16 core for $g=0$ where we can observe that the computations have been performed by an adaptative meshing process, showing a higher number of triangles at some regions, especially close to the angles of the ferrite material. In Figure 2(b) we plot the flux lines in the core. As can be seen, the $B$ lines are closed $(\nabla B=0)$ and contained inside the core because the relative permeability of the ferrite is very high compared to the one of vacuum $\left(\mu_{r}>>1\right)$. We used B (in bold) to denote the magnetic field vector.

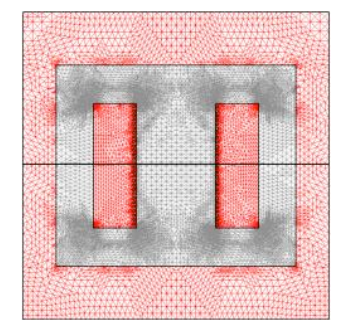

(a)

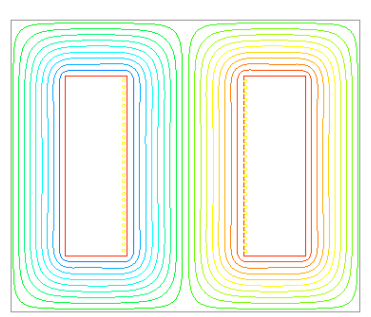

(b)
Fig. 2. (a) Mesh generated by the simulation program using a $2 \mathrm{D}$ domain with $g=0$ and $\tau=1 \%$. (b) Flux lines in the core.

As an example, in Figure 3 we plot the modulus of the induction and of the magnetic field for different excitation currents (linear, intermediate and saturation regions) in the E47/20/16 core for $g=0$ along the path drawn (line) in Figure 2(a). The center of the core is located at $x=30 \mathrm{~mm}$.
As we can see, the magnetic field $|\mathrm{B}|$ is uniform inside the core and rises with increasing current. We can appreciate discontinuities of $|B|$ at the ferrite-air interfaces. Similarly, the value of $|\mathrm{H}|$ increases with the current. Regarding the experimental validation, as the 3D simulation does not converge, we only present the comparison between results obtained by $2 \mathrm{D}$ simulations and by experiment. In Figure 4 we show the $\Phi-/$ curves and $L-/$ curves for the biggest core $(E 65 / 32 / 27)$ where we can see good agreement between the results.

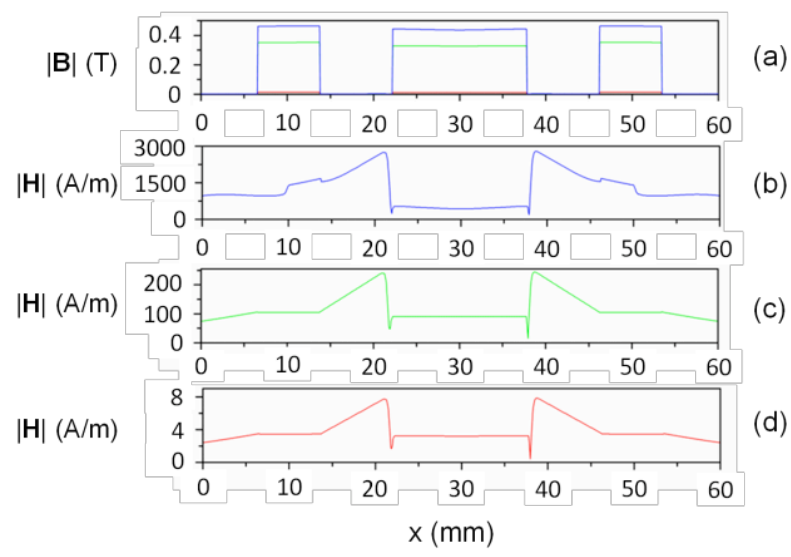

Fig. 3. (a) Moduli of the B vector for $I=0.01(-), 0.3(-)$ and 3 $\mathrm{A}(-)$ and moduli of the $\mathrm{H}$ vector for (b) $3,(-),(c) 0.3(-)$ and (d) $0.01 \mathrm{~A}(-)$.

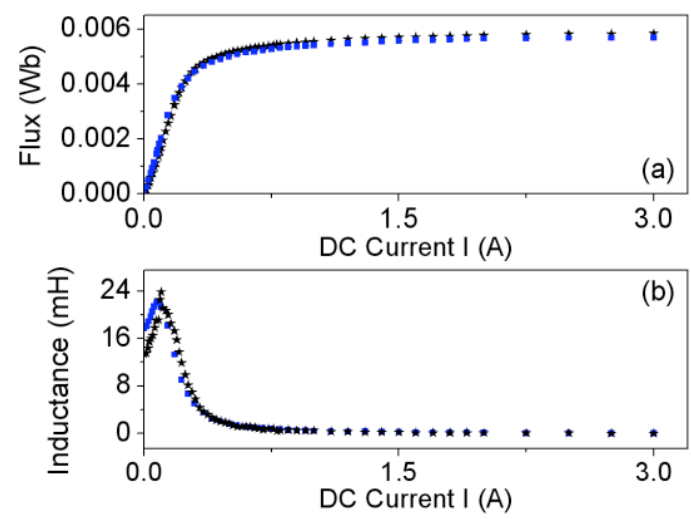

Fig. 4. (a) $\Phi$-/ curves (b) $L-/$ curves for the $(E 65 / 32 / 27)$ obtained by experiment $(-*-)$ and by $2 \mathrm{D}$ simulations $(-\square-)$.

Since for the smallest core (E20/10/5) there was convergence both in the $2 \mathrm{D}$ and $3 \mathrm{D}$ simulations, we present the comparison of the results of the simulations for this core. In Table 6 we show the computational cost (CPU time and number of finite elements) in the case of an ungapped core $(g=0 \mu \mathrm{m})$ for two values of the DC excitation current, $/=$ $0.01 \mathrm{~A}$ (linear region) and $/=20 \mathrm{~A}$ (saturation region). We have carried out the $3 \mathrm{D}$ simulations in the cylindrical domain $D:(r, Z) \in[0,14] \times[-15,15]$ and the $2 \mathrm{D}$ simulations in the corresponding cross-section.

Table 6. Computational cost for $\tau=1 \%$ and $g=0 \mu \mathrm{m}$.

\begin{tabular}{|c|c|c|c|c|}
\hline & \multicolumn{2}{|c|}{ CPU time } & \multicolumn{2}{c|}{ Elements } \\
\hline$I(\mathrm{~A})$ & $2 \mathrm{D}(\mathrm{s})$ & $3 \mathrm{D}$ & $2 \mathrm{D}$ & $3 \mathrm{D}$ \\
\hline 0.01 & 2 & $3 \mathrm{~min}$. & 5894 & 77280 \\
\hline 20 & 2 & $11 \mathrm{~min} .46 \mathrm{~s}$ & 5894 & 87327 \\
\hline
\end{tabular}


We can see that the computational cost is in favor of the 2D simulation, being here of the order of a few seconds and in the $3 \mathrm{D}$ simulations of the order of minutes. In $3 \mathrm{D}$ the CPU time for the excitation current in the linear region is lower than in the other regions (intermediate and saturation regions) while in the case of $2 \mathrm{D}$, the CPU time is identical. In addition, for this particular case, the number of elements in $3 \mathrm{D}$ is not constant for all values of the excitation current due to the nature of the adaptative mesh, while in the simulations in 2D it remains constant for both values of $l$.

Regarding the experimental validation, in Figure 5 we show the results of the flux and the inductance obtained by 3D and 2D FEA and compare them with the ones obtained experimentally for the specific E20/10/5 core with 15 turns. There is good agreement between the results giving the $2 \mathrm{D}$ model quite correct results with the advantages of simplification, low computational cost and easy calculation procedure, when compared to other procedures. Consequently, we think that a 3D model is not really useful in this kind of geometry. The slight differences in 2D could be due to the way of computing $\Phi$ evaluating the line integral of the $B_{y}$ field (instead of evaluating the surface integral) and supposing that $B_{y}$ is constant in the direction of the core thickness.

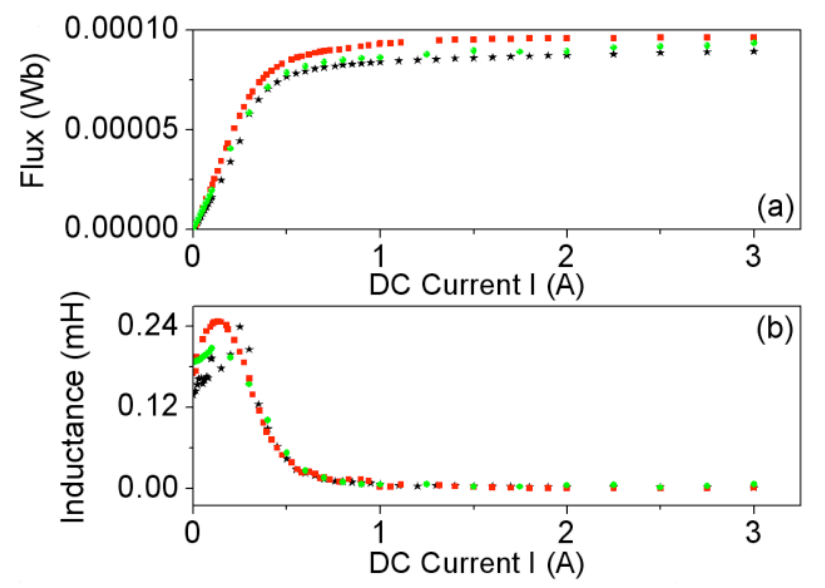

Fig. 5. Results for the E20/15/10 core with 15 turns. (a) $\Phi-/$ curves. (b) $L-/$ curves. Experiment $(-*-)$ and $3 \mathrm{D}\left(-{ }^{-}\right)$and $2 \mathrm{D}$ simulations $(-\square-)$ with $\mathrm{g}=5 \mu \mathrm{m}$.

\section{Experimental study of the influence of the number of turns on the flux and inductance}

To complete the results we have studied experimentally the influence of the number of turns of the inductor on the values of $\Phi$ and $L$ and on the $B$ and $H$ fields. For this purpose, we study three inductors with identical E34/14/9 core but with a different number of turns (18, 28 and 38). We built three two-winding transformers, one for each case, and took measurements the same way we did in the measuring procedure (section 2).

In Figure 6 we show the results of the measured $\Phi$-/ and $L-/$ curves. It can be observed that in order to reach the saturation value of the material for low excitation currents, it is necessary to increase the number of turns. As is expected, the magnetic flux is proportional to the number of turns. The saturation value of the inductance and the values of $B$ and $H$ are independent of the number of turns. The $L-/$ curves reach their maximum at the same current intensity, independent of the number of turns. These maximums are reached at $I=$ $0.05 \mathrm{~A}$, and have a value of $0.5 \mathrm{mH}$ for 18 turns, $1.5 \mathrm{mH}$ for 28 turns and $2.5 \mathrm{mH}$ in the case of 38 turns.

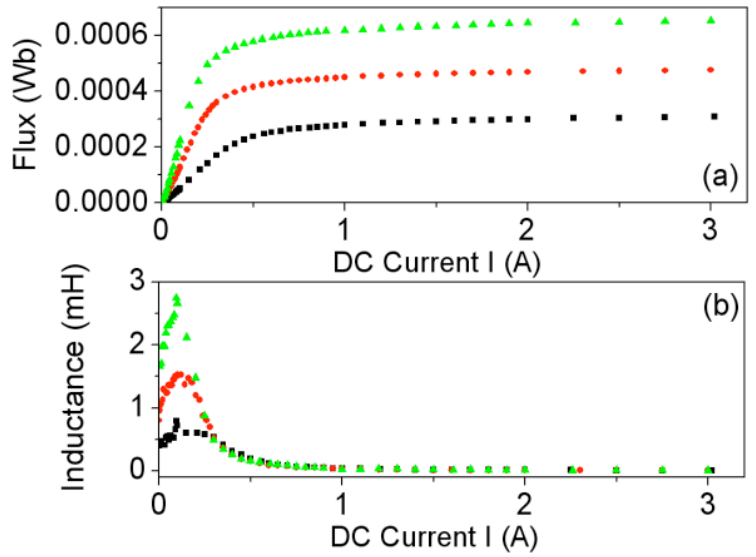

Fig. 6. (a) $\Phi-I$ curves and (b) $L-I$ curves measured with the E34/14/9 core for: 18 turns (--), 28 turns (-๑) and 38 turns $(-\Delta-)$.

\section{Conclusions}

We have presented a method to compute the inductance (the parameter of the equivalent electrical circuit of an inductor). We have explored the behavior of four E-type ferrite inductors under DC excitation currents from the linear to the saturation regions and we have observed that while in $3 \mathrm{D}$ there was only convergence with the smallest core; in 2D we achieved convergence for all sizes, excitation current values and gap-thicknesses. By using a cross-section of the real inductor we are able to reproduce with precision the inductance curves from the linear to the saturation regions. Possible future work would be to include the effect of power losses on this type of cores.

\section{References}

1. N. Mohan, T. M. Undeland, W. P. Robbins, Power Electronics: Converters, Applications, and Design (John Wiley and Sons, Inc., $3^{\text {rd }}$ edition, 2006)

2. E. C. Snelling, Soft Ferrites, Properties and Applications (Butterworths, $2^{\text {nd }}$ edition, 1988)

3. P. R. Wilson, J. N. Ross, A. D. Brown, IEEE Trans. Power Electron. 17 (2002)

4. P. Nakmahachalasint, K. D. T. Ngo, L. Vu-Quoc, IEEE Trans. Power Electron. 17 (2002)

5. A. Benabou, S. Clénet, F. Piriou, J. Magn. Magn. Mater. 261 (2003)

6. R. Prieto, J. A. Cobos, O. García, P. Alou, J. Uceda, IEEE Trans. Ind. Electron. 50 (2003)

7. A. Standler, M. Albach, IEEE Trans. Magn. 42 (2006) 